\title{
SEMILOCAL ANALYSIS OF EQUATIONS WITH SMOOTH OPERATORS
}

\author{
GEORGE J. MIEL \\ Department of Mathematical Sciences \\ University of Nevada \\ Las Vegas, Nevada 89154 U.S.A. \\ (Received September 2, 1980)
}

ABSTRACT. Recent work on eemilocal analysis of nonlinear operator equations is informally reviewed. A refined version of the Kantorovich theorem for Newton's method, with new error bounds, is presented. Related topics are briefly surveyed. KEY WORDS AND PHRASES. Nonlinear equations, semilocal theorem, Kantorovich theorem, Newton method.

1980 MATHEMATICS SUBJECT CLASSIFICATION CODES. 65H10, 65J15, $47 \mathrm{H} 17$.

1. INTRODUCTION.

In the terminology of Ortega-Rheinholdt [15], a semilocal analysis for a given equation $F x=0$ establishes the existence of a local solution $x^{*}$, by showing that a sequence of approximate solutions $x_{n}$ converges to $x^{*}$, and it also yields computable bounds for the errors $\left\|x^{*}-x_{n}\right\|$. The operator $F$ is generally assumed to be Fréchet differentiable and the basic idea is to take each $x_{n}$ as the solution of an approximating linear operator equation. The central result of this kind is the Kantorovich theorem for Newton's method. Most other semilocal results are related to that famous theorem, since they involve linearization processes based on approximate derivatives. The theory can be used to establish the existence and uniqueness of solutions for specific equations without finding the solutions. Unfortunately, its application to real computation is fraught with difficulties. Our purpose here is to describe research which attempts to bring semilocal theory 
a bit closer to the computer. We will do this in two ways. First, we will state and prove a refined version of the Kantorovich theorem, which includes new error bounds. Secondly, we will give a brief and informal survey of related topics, with a view of bringing out the benefits and drawbacks of semilocal analyses.

\section{THE KANTOROVICH THEOREM.}

Let $X$ and $Y$ be Banach spaces and let $D$ be an open convex subset of $X$. The open ball $\left\{x:\left\|x-x_{0}\right\|<r\right\}$ and its closure are denoted by $S\left(x_{0}, r\right)$ and $\bar{S}\left(x_{0}, r\right)$ respectively.

THEOREM. Let $F: D \subset X \rightarrow Y$ be Fréchet differentiable. Assume that $\mathrm{F}^{\prime}\left(\mathrm{x}_{0}\right)$ is invertible for some $x_{0} \varepsilon D$, and that

$$
\begin{aligned}
& \left\|F^{\prime}\left(x_{0}\right)^{-1} F x_{0}\right\| \leq a, \\
& \left\|F^{\prime}\left(x_{0}\right)^{-1}\left(F^{\prime}(x)-F^{\prime}(y)\right)\right\| \leq k\|x-y\|, x, y \in D, \\
& \bar{S}\left(x_{0}, t^{*}\right) \subset D, \quad h=2 K a \leq 1, \quad t^{*}=(1-\sqrt{1-h}) / K .
\end{aligned}
$$

Then

i) $F^{\prime}(x)$ is invertible for every $x \varepsilon S\left(x_{0}, t^{*}\right)$.

ii) The iterates $x_{n+1}=x_{n}-F^{\prime}\left(x_{n}\right)^{-1} F x_{n}$ remain in $S\left(x_{0}, t^{*}\right)$ and converge to a root $\mathrm{x}^{*}$ of $\mathrm{F}$.

iii) The root $x^{*}$ is unique in $D \cap S\left(x_{0}, t^{* *}\right), t^{* *}=(1+\sqrt{1-h}) / K$.

Moreover, if

$$
\begin{aligned}
& g(t)=\frac{K}{2} t^{2}-t+a=\frac{K}{2}\left(t^{*}-t\right)\left(t^{* *}-t\right), \\
& t_{0}=0, t_{n+1}=t_{n}-g^{\prime}\left(t_{n}\right)^{-1} g\left(t_{n}\right),
\end{aligned}
$$

then

iv)

v)

$$
\begin{gathered}
\left\|x^{*}-x_{n+1}\right\| \leq a_{n}\left\|x^{*}-x_{n}\right\|^{2}, a_{n}=\frac{t^{*}-t_{n+1}}{\left(t^{*}-t_{n}\right)^{2}}, \\
\left\|x^{*}-x_{n}\right\| \leq \frac{t^{*}-t_{n}}{\left(t_{n}-t_{n-1}\right)^{\mu}}\left\|x_{n}-x_{n-1}\right\|^{\mu} \leq \frac{t^{*}-t_{n}}{t_{1}^{\mu}}\left\|x_{1}-x_{0}\right\|^{\mu},
\end{gathered}
$$

for every $\mu \varepsilon[0,2]$. 
vi) $\frac{2\left\|x_{n+1}-x_{n}\right\|}{1+\sqrt{1+4\left(\frac{t_{n+1}-t_{n}}{t_{n}-t_{n-1}}\right)^{2}}} \leq \frac{1}{2 a_{n}}\left(\sqrt{1+4 a_{n}}\left\|x_{n+1}-x_{n}\right\|-1\right) \leq\left\|x^{*}-x_{n}\right\|$.

Also, the uniqueness statement (iii) and the bounds in (iv), (v), (vi) are best possible.

PROOF. By invariance of Newton iterates under affine transformations,

$$
\begin{gathered}
x_{n+1}=x_{n}-G^{\prime}\left(x_{n}\right)^{-1} G x_{n}, \\
G x=F^{\prime}\left(x_{0}\right)^{-1} F x .
\end{gathered}
$$

The Banach lemma yields (i). If both $x, H x=x-G^{\prime}(x)^{-1} G x \in S\left(x_{0}, t^{*}\right)$ then

$$
\|\mathrm{H}(\mathrm{H} \mathbf{x})-\mathrm{H} \mathbf{x}\| \leq \frac{\mathrm{K} / 2}{1-\mathrm{K}\left\|\mathrm{H}-\mathrm{x}_{0}\right\|}\|\mathrm{Hx}-\mathbf{x}\|^{2} \text {. }
$$

The sequence $\left\{t_{n}\right\}$ satisfies the conditions $\left\|x_{1}-x_{0}\right\| \leq t_{1}=a, t_{n-1}<t_{n}, l i m t_{n}=t^{*}$, and

$$
\frac{K / 2}{1-K t_{n}}=\frac{t_{n+1}-t_{n}}{\left(t_{n}-t_{n-1}\right)^{2}}
$$

An induction argument shows that $\left\{x_{n}\right\}$ exists and that $\left\{t_{n}\right\}$ is a majorizing sequence. Statement (ii) follows; the continuity at $\mathrm{x}^{*}$ of $\mathrm{F}^{\prime}$ implies that $\mathrm{Fx}^{*}=0$. Statement (iii) follows by consideration of the simplified Newton method. Lettin'T $e_{n}=t^{*}-t_{n}$, we have

$$
\begin{gathered}
e_{0}=t^{*}, \quad e_{n+1}=\frac{e_{n}^{2}}{2 e_{n}+\Delta}, \Delta=t^{* *}-t^{*}, \\
\left\|G^{\prime}\left(x_{n}\right)^{-1}\right\| \leq-g^{\prime}\left(t_{n}\right)^{-1}=\frac{1}{1-K t_{n}}=\frac{2}{k} \cdot \frac{e_{n+1}}{e_{n}^{2}} .
\end{gathered}
$$

Use (2.5) and a mean-value theorem on

$$
\left\|x^{*}-x_{n+1}\right\| \leq\left\|G^{\prime}\left(x_{n}\right)^{-1}\right\|\left\|G x^{*}-G x_{n}-G^{\prime}\left(x_{n}\right)\left(x^{*}-x_{n}\right)\right\|
$$

to get (iv). Use (2.2) and $(2.3)$ as in [11] to derive (v).

From the identity $x_{n+1}-x_{n}=\left(x^{*}-x_{n}\right)+G^{\prime}\left(x_{n}\right)^{-1}\left(G x^{*}-G x_{n}-G^{\prime}\left(x_{n}\right)\left(x^{*}-x_{n}\right)\right)$ and (2.5), we get

$$
\frac{e_{n+1}}{e_{n}^{2}}\left\|x^{*}-x_{n}\right\|^{2}+\left\|x^{*}-x_{n}\right\|-\left\|x_{n+1}-x_{n}\right\| \geq 0
$$

The sharper lower bound in (vi) follows. Bring the square root in that bound to 
the denominator, use $\left\|x_{n+1}-x_{n}\right\| \leq t_{n+1}-t_{n}$ and

$$
\frac{e_{n+1}}{e_{n}^{2}}=\frac{t_{n+1}-t_{n}}{\left(t_{n}-t_{n-1}\right)^{2}}
$$

which results from (2.3) and (2.5), to get the other lower bound. Take $F=g$, $x_{0}=t_{0}$ to obtain the optimality of (iii), (iv), (v). The weaker optimality of (vi) is obtained by constructing a scalar function as in [5]. This completes the proof.

We discuss features of the above version of the Kantorovich theorem:

1) The theorem is affine invariant and the transformation (2.1) is an optimal scaling [4]. The parameter $h$ may be considered a measure of the nonlinearity of $\mathrm{F}$.

2) The estimates given in (iv) appear to be new. They show that the majorizing sequence yields not only second r-order convergence, but the stronger second q-order as well. Indeed, if $h<1$ then $(2.4)$ shows that $\lim a_{n}=1 / \Delta<\infty$.

3) The upper bounds in (v) were derived by Miel [11]. The bound with $\left\|x_{n}-x_{n-1}\right\|^{\mu}$ is usually considerably better than the one with $\left\|x_{1}-x_{0}\right\|^{\mu}$. Both bounds are monotone decreasing functions of $\mu$. The well known upper bounds of Gragg-Tapia [5] correspond to the case $\mu=1$. The bound with $\left\|x_{n}-x_{n-1}\right\|^{2}$ is sharper. We prove below that it is also sharper than the upper bound of Potra-Pták [17].

4) Statement (vi) gives an improvement of the lower bound of Gragg-Tapia [5], since the latter can be shown to be equivalent to the left-most expression. 5) The bounds are expressed in terms of the majorizing sequence, but since Newton iterates for quadratic polynomials are known in closed form $[9$, p. 28] or [16, Appendix F], these bounds can be given explicitly .

2.1. NONDISCRETE INDUCTION.

Like the principle of majorants, this technique developed by Pták $[20,21,22]$ yields simultaneously convergence and computable error bounds. We briefly describe the method. Let $\mathrm{H}: \mathrm{X} \rightarrow \mathrm{X}$ and consider the iteration

$$
\mathrm{x}_{0} \varepsilon \mathrm{X}, \mathrm{x}_{\mathrm{n}}=\mathrm{H} \mathrm{x}_{\mathrm{n}-1}
$$


Let $T$ denote an open interval contained in $(0, \infty)$. The following lemma, whose proof can be found in [17], is a special case of the Induction Theorem of Pták [20].

LEMMA. Assume that there exists

1) a map $w: T \rightarrow T$ such that the series

$$
\sigma(r)=r+\omega(r)+\omega(\omega(r))+\ldots+\omega^{n}(r)+\ldots
$$

converges for every $r \in T$,

2) a family of sets $Z(r) \subset X, r \varepsilon T$, such that

$$
\begin{gathered}
x_{0} \in z\left(x_{0}\right) \text { for some } r_{0} \in T \\
F(x) \in \bar{S}(x, r) \cap Z(\omega(r)) \text { whenever } r \in T \text { and } x \in Z(r)
\end{gathered}
$$

Then the iterates (2.6) converge to some $x^{\star} \varepsilon x$ and

$$
\left\|x^{*}-x_{n}\right\| \leq \sigma\left(\omega^{n}\left(r_{0}\right)\right) \text {. }
$$

If $x_{n-1} \varepsilon z\left(a_{n}\right), a_{n}=\left\|x_{n}-x_{n-1}\right\|$, then the error bounds

$$
\left\|x^{*}-x_{n}\right\| \leq \sigma\left(a_{n}\right)-a_{n}
$$

are also valid.

With the notation here, the Kantorovich theorem can be established by taking

$$
\omega(r)=\frac{r^{2}}{2 \sqrt{r^{2}+\Delta 44}},
$$

and $Z(x)$ the set of elements $x \in X$ such that

$$
\begin{aligned}
& \left\|x-x_{0}\right\| \leq \sigma(a)-\sigma(r), \\
& F^{\prime}(x)^{-1} \text { exists, } \\
& \inf \left\|F^{\prime}(x) y\right\| \geq b^{-1}-K[\sigma(a)-\sigma(x)], \\
& \|y\|=1 \\
& \left\|F^{\prime}(x)^{-1} F x\right\| \leq r .
\end{aligned}
$$

Pták [21] thus derived the a priori upper bounds of Gragg-Tapia with the use of (2.7), and recently, Potra-Pták [17] used (2.8) to obtain a posteriori bounds sharper than those of Gragg-Tapia.

The Potra-Pták bounds are

$$
\gamma\left(\Delta / 2,\left\|x_{n+1}-x_{n}\right\|\right) \leq\left\|x^{*}-x_{n}\right\| \leq \delta\left(\Delta / 2,\left\|x_{n}-x_{n-1}\right\|\right),
$$




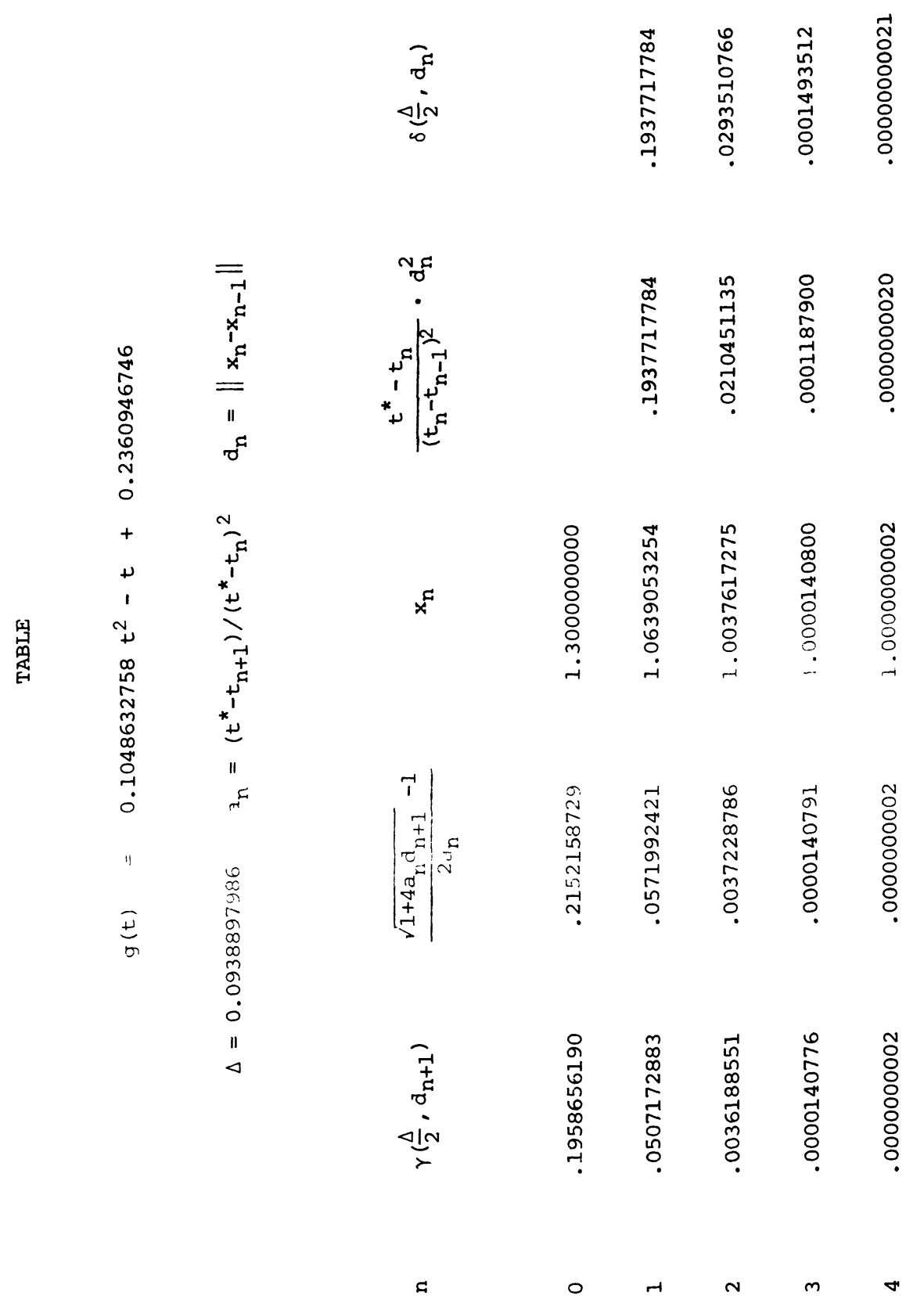




$$
\begin{gathered}
\gamma(s, t)=\left(s^{2}+4 t^{2}+4 t\left(s^{2}+t^{2}\right)^{\frac{1}{2}}\right)^{\frac{1}{2}}-\left(t+\left(s^{2}+t^{2}\right)^{\frac{1}{2}}\right), \\
\delta(s, t)=\left(s^{2}+t^{2}\right)^{\frac{1}{2}}-s .
\end{gathered}
$$

The table compares these bounds with (v) and (vi) for the scalar cubic $F(x)=\frac{1}{3}$ $\left(x^{3}-1\right)$, with $x_{0}=1.3, a=0.236095, k=0.209727$. The bounds generated by the majorizing sequence are seen to be finer. We prove that $\left(t^{*}-t_{n}\right) \cdot d_{n}^{2} /\left(t_{n}-t_{n-1}\right)^{2}$ is generally sharper than $\delta\left(\Delta / 2, d_{n}\right)$. We have

$$
\delta(s, t)=\epsilon(s, t) \cdot t^{2}, \epsilon(s, t)=1 /\left(\left(s^{2}+t^{2}\right)^{\frac{1}{2}}+s\right) .
$$

Use $t_{n}-t_{n-1}=e_{n-1}-e_{n}$ and (2.4) to get

$$
\frac{t^{*}-t_{n}}{\left(t_{n}-t_{n-1}\right)^{2}}=\epsilon\left(\frac{\Delta}{2}, t_{n}-t_{n-1}\right) \leq \epsilon\left(\frac{\Delta}{2},\left\|x_{n}-x_{n-1}\right\|\right)
$$

for the inequality, use that $\epsilon(s, t)$ is monotone decreasing in $t$.

\section{INFORMAL DISCUSSION.}

In this section, we give a short list of references and we consider benefits and drawbacks of semilocal analyses.

\subsection{SOME REFERENCES.}

A history of the Kantorovich theorem and some of its relatives is given in Ortega-Rheinboldt [15, p. 428] and in Ostrowski [16, p. 404]. There must be dozens, if not hundreds, of semilocal theorems for nonlinear operator equations, and it is impossible to attempt here a comprehensive list of references. With a disclaimer for omissions, we mention references for representative results. Krasnosel'skii and Rutitskii studied a class of Newton-type iterative processes; equations with nondifferentiable operators were treated by Zinchenko and also Kusakin; see [8, Bibliography] for the references.

A refinement of the majorant technique was used by Ortega [14] to present an elegant proof of the Kantorovich theorem, and more generally, by Rheinboldt [25], to establish a general semilocal theory for iteration of the form,

$$
\mathrm{x}_{\mathrm{n}+1}=\mathrm{x}_{\mathrm{n}}-\mathrm{D}\left(\mathrm{x}_{\mathrm{n}}\right)^{-1} \mathrm{~F} \mathrm{x}_{\mathrm{n}}
$$

where $D(x)$ is an approximate derivative of $F$ satisfying certain conditions. The 
corresponding majorizing sequence is generated by

$$
t_{0}=0, t_{n+1}=t_{n}-d\left(t_{n}\right)^{-1} f\left(t_{n}\right),
$$

where $d$ and $f$ are respectively a linear and a quadratic polynomial. These scalar functions satisfy the convergence conditions for a subclass of methods, including Newton's method when $D(x)=F^{\prime}(x)$, and (3.2) then becomes a special case of (3.1) with $F=f, D=d, x_{0}=t_{0}$. Miel $[10,11]$ showed that under the hypotheses of Rheinboldt's theorem, the majorizing sequence yields

$$
\left\|x^{*}-x_{n}\right\| \leq \frac{t^{*}-t_{n}}{\left(t_{n}-t_{n-1}\right)^{\mu}}\left\|x_{n}-x_{n-1}\right\|^{\mu} \leq \frac{t^{*}-t_{n}}{t_{1}^{\mu}}\left\|x_{1}-x_{0}\right\|^{\mu}, 0 \leq \mu \leq 1 .
$$

These error bounds are clearly optimal for the proper subclass of methods. For the Newton method, as shown in the previous section, the stronger statement with $0 \leq \mu \leq 2$ is valid.

Rheinboldt's hypotheses on $D(x)$ in the semilocal analysis of (3.1) turn out to be restrictive; Dennis [1] used a majorizing sequence to extend the result for methods which have approximate derivatives of bounded deterioration, and thus include certain generalized secant algorithms. Consideration of these algorithms led to the research on so-called quasi-Newton methods, surveyed in $[2,3]$. Potra-Pták $[17,18,19]$ used nondiscrete induction to obtain convergence and error bounds for the Newton, multistep Newton, and generalized regular falsi methods. We proved that their upper bound for Newton's method is related to, but not as sharp as the finer bound in (3.3) with $\mu=2$.

\subsection{ADVANTAGES.}

Benefits gained from the Kantorovich theorem and related semilocal theorems are summarized below:

1) One can establish domains of existence and uniqueness for a solution of a nonlinear operator equation, with no actual knowledge of the solution.

2) A constructive method for approximating such a solution is provided, consisting of a convergent sequence of solutions of linearized operator equations.

3) A domain of attraction $S$ is established with the property that if the 
iterates reach $\mathbf{S}$ then they will stay in $\mathbf{S}$ and converge to a solution.

4) Error bounds are available provided that one can evaluate the constants involved in the hypotheses of the theorem.

5) Newton's method is self-corrective: $x_{n+1}$ depends only of $F$ and $x_{n}$ ' so that errors from previous iterates do no propagate.

Property (5) is an advantage of Newton's method which is not shared by quasiNewton methods. We include (4) as an advantage despite the warnings in [1,2] against the use of majorizing sequences for getting error bounds. The reasons cited were the apparent $r$-order of convergence, the coarseness of the bounds, and the difficulty in calculating the required constants. Because of results which partially overcome these objections, the views against majorizing sequences should perhaps be re-evaluated. It was shown in the last section that the majorizing sequence for Newton's method does imply q-quadratic convergence and that the bound with $\left\|x_{n}-x_{n-1}\right\|^{2}$ is sharper than the usual ones with $\left\|x_{n}-x_{n-1}\right\|$ and $\left\|x_{1}-x_{0}\right\|$. The problems associated with the local nature of the estimates and the verification of hypotheses, however, do remain. In this connection, we point to research on computer verification of semilocal conditions by interval analysis $[12,13,23]$

\subsection{DISADVANTAGES.}

From a practical aspect, statement (2) above is no panacea to the numerical analyst: each linear operator equation in the constructive process must still be reduced to a computable form. This brings us to the first drawback in our 1 ist.

1) The theory does not provide a means of discretizing an operator equation into a corresponding finite system of equations.

2) Stringent hypothese require that the iterates be in the vicinity of a root before a theorem will guarantee convergence and provide error bounds.

3) The computation of the constants in these hypotheses, especially the Lipschitz constants, is difficult.

4) Newton's method requires a new Fréchet derivative $F^{\prime}\left(x_{n}\right)$ at each step $n$. 
5) A system of linear equations must be solved at each step. For Newton's method in dimension $N$, this requires a costly $O\left(N^{3}\right)$ arithmetic operations.

With respect to (4), it should be noted that derivatives have been compiled by suitable software as easily as functions $[7,24]$. The research on quasi-Newton methods is motivated by (4) and (5). These methods use ingenious approximate Jacobians to avoid the evaluation of $F^{\prime}(x)$ and to reduce from $O\left(N^{3}\right)$ to $O\left(N^{2}\right)$ the cost in the solution of linear systems. The price paid is a reduction from second order to superlinear convergence. Local analysis of quasi-Newton algorithms has made two fundamental contributions to the theory of iterative methods: the notion of bounded deterioration of approximate derivatives and a characterization of $q$-superlinear convergence. The algorithms have been studied extensively for optimization problems $[2,3]$.

ACKNOWLEDGEMENT. This paper was presented at the $\mathrm{V}$-th International Conference on Operator Theory held June 1980 in Timisoara, Romania.

\section{REFERENCES}

1. DENNIS, J.E. Toward a Unified Convergence Theory for Newton-1ike Methods, in Nonlinear Functional Analysis and Applications, L.B. Rall, Ed., Academic Press, New York, 1971.

2. DENNIS, J.E. A Brief Introduction to Quasi-Newton Methods, in Numerical Analysis, G.H. Golub and J. Oliger, Eds., Proc. Sympos. Appl. Math. 22, Amer. Math. Soc., Providence, R.I., 1978.

3. DENNIS, J.E. and MORÉ, J.J. Quasi-Newton Methods, Motivation and Theory, SIAM Rev. 19 (1977), 46-89.

4. DEUFLHARD, P. and HEINDL, G. Affine Invariant Convergence Theorems for Newton's Method and Extensions to Related Methods, SIAM J. Numer. Anal. 16 (1979), $1-10$.

5. GRAGG, W.B. and TAPIA, R.A. Optimal Error Bounds for the Newton-Kantorovich Theorem, SIAM J. Numer. Anal. 11 (1974), 10-13.

6. KANTOROVICH, L.V. Functional Analysis and Applied Mathematics, Uspehi Mat. Nauk 3 (1948), 89-185 (Russian); English transl., Rep. 1509, National Bureau of Standards, Washington, D.C., 1952.

7. KEDEM, G. Automatic Differentation of Computer Programs, ACM Trans. Math. Software 6 (1980), 150-165.

8. KRASNOSEL'SKII, M.A. ET AL, Approximate Solution of Operator Equations, translated from Russian by D. Louvish, Wolters-Noordhoff, Groningen, 1972. 
9. LYUSTERNIK, L.A. ET AL, Handbook for Computing Elementary Functions, transslated from Russian by G.J. Tee, Pergamon Press, New York, 1965.

10. MIEL, G.J. Unified Error Analysis for Newton-type Methods, Numer. Math. 33 (1979), 391-396.

11. MIEL, G.J. Majorizing Sequences and Error Bounds for Iterative Methods, Math. Comp. 34 (1980), 185-202.

12. MOORE, R.E. A Test for Existence of Solutions to Nonlinear Systems, SIAM J. Numer. Anal. 14 (1977), 1051-1065.

13. MOORE, R.E. New Results on Nonlinear Systems, in Interval Mathematics 1980, Edited by K. Nickel, Academic Press, New York, 1980.

14. ORTEGA, J.M. The Newton-Kantorovich Theorem, Amer. Math. Monthly 75 (1968) 658-660.

15. ORTEGA, J.M. and RHEINBOLDT, W.C. Iterative Solution of Nonlinear Equations in Several Variables, Academic Press, New York, 1970.

16. OSTROWSKI, A.M. Solution of Equations in Euclidean and Banach Spaces, Academic Press, New York, 1973.

17. POTRA, F.A. and PTÁ, V. Sharp Error Bounds for Newton's Process, Numer. Math. 34 (1980), 63-72.

18. POTRA, F.A. and PTAK, V. On a Class of Modified Newton Processes, Preprint Series in Mathematics No. 59, INCREST, Bucharest, 1979.

19. POTRA, F.A. and PTÁK, V. A Generalisation of Regular Falsi, Preprint Series in Mathematics No. 10, INCREST, Bucharest, 1980.

20. PTÁK, V. Nondiscrete Mathematical Induction and Iterative Existence Proofs, Linear Algebra and its Applications 13 (1976), 223-236.

21. PTÁK, V. The Rate of Convergence of Newton's Process, Numer. Math. 25 (1976), 279-285.

22. PTÁK, V. Nondiscrete Mathematical Induction, in General Topology and its Relations to Modern Analysis and Algebra IV, Lecture Notes in Mathematics 609, Springer Verlag, 1977.

23. RALL, L.B. A Comparison of the Existence Theorems of Kantorovich and Moore, SIAM J. Numer. Anal. 17 (1980), 148-161.

24. RALL, L.B. Applications of Software for Automatic Differentiation in Numerical Computation, Computing, Suppl. 2 (1980), 141-156.

25. RHEINBOLDT, W.C. A Unified Convergence Theory for a Class of Iterative Process, SIAM J. Numer. Anal. 5 (1968), 42-63. 


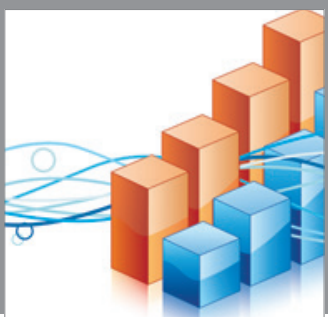

Advances in

Operations Research

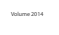

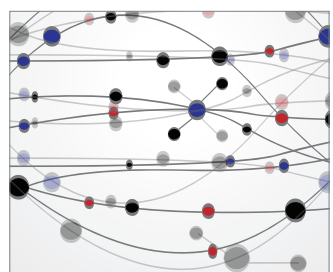

\section{The Scientific} World Journal
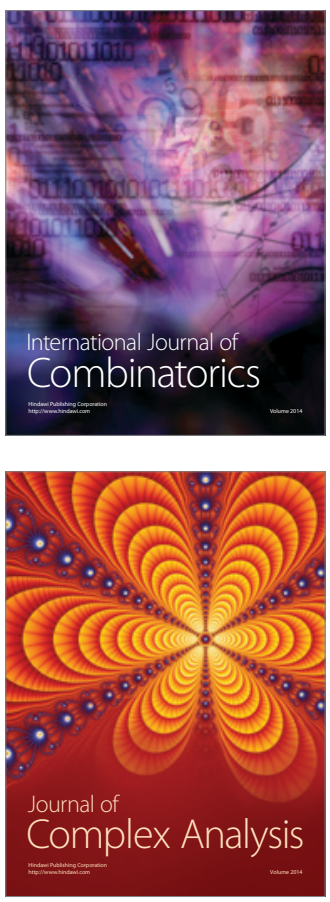

International Journal of

Mathematics and

Mathematical

Sciences
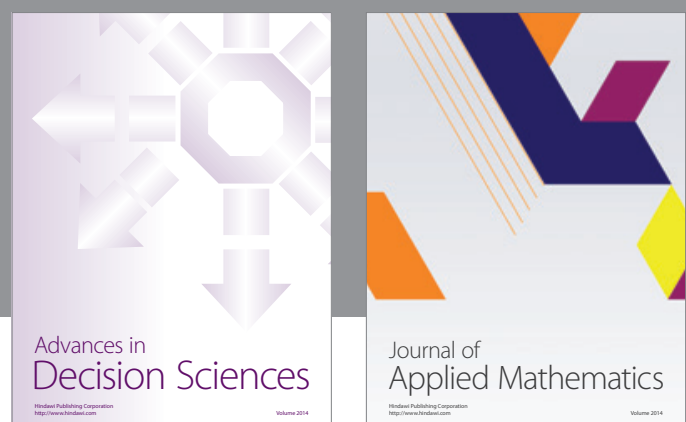

Journal of

Applied Mathematics
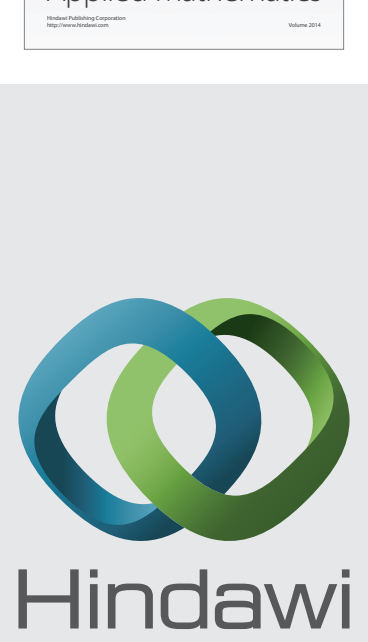

Submit your manuscripts at http://www.hindawi.com
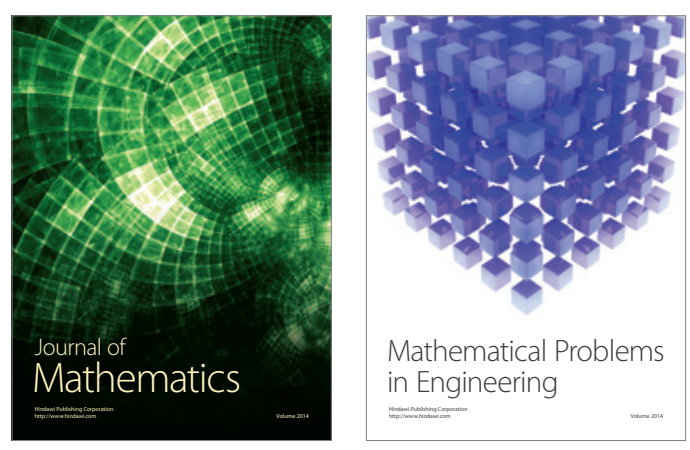

Mathematical Problems in Engineering
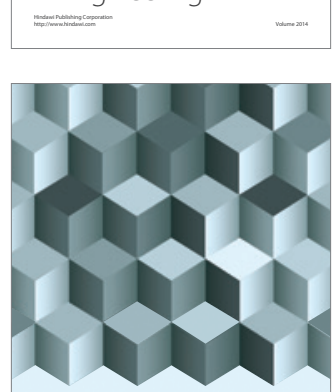

Journal of

Function Spaces
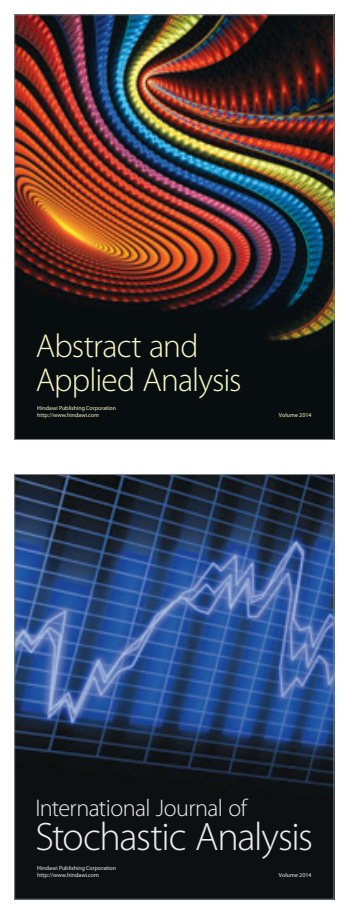

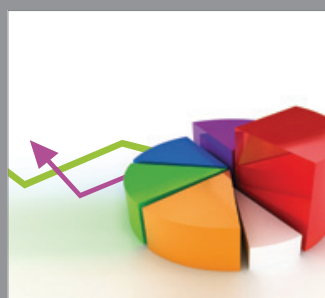

ournal of

Probability and Statistics

Promensencen
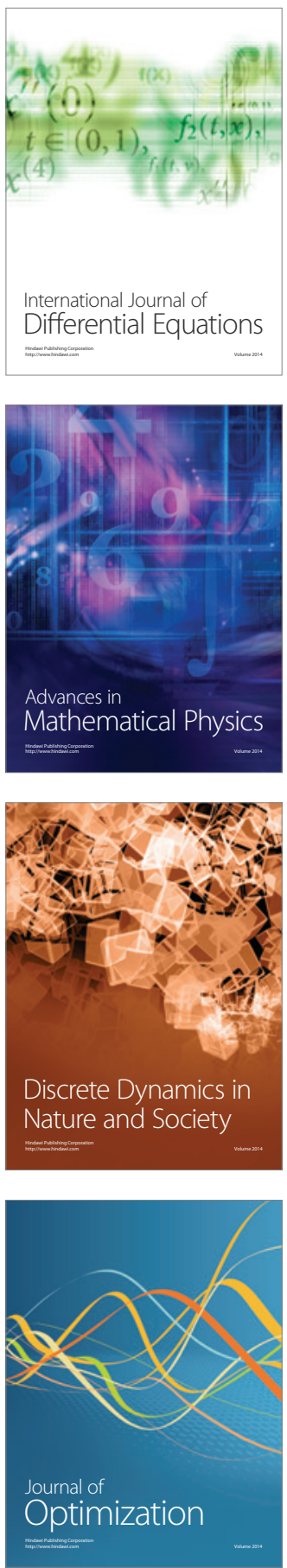